\section{Inadequate Seizures in ECT}

SIR: The question of inadequate seizures in ECT mentioned by Jones (Journal, August 1988, 153, 264 $265)$ seems to have arisen in recent years. Prior to 1980 most treatments were given with a sine wave stimulus from constant current apparatus, and the clinical results were good. Many achieved over $90 \%$ recovery rates with small numbers of treatments. An unjustified outcry concerning "side-effects" of ECT then arose, leading to a concentration on reducing "side-effects" which was at the expense of clinical effectiveness. Unfortunately, the development of constant current pulse-type stimuli at that time enhanced the problem, since they were more efficient in producing seizures with a much lower dosage and also reduced the side-effects.

It was firmly believed that the clinical effect would always be present provided that a seizure was produced. It is now clear that a larger stimulus than that required to produce a seizure is necessary to ensure a good clinical response.

Although seizures can be produced in many patients with a stimulus dose as low as $150 \mathrm{mC}$, most require at least $275 \mathrm{mC}$ to give a good clinical effect, and a few cases require a dose of $375 \mathrm{mC}$. (It should be remembered that the old sine wave instruments would deliver $800 \mathrm{mC}$ or more to patients with a low resistance.) These dosages apply to bilateral treatment, when approximately $5 \%$ of the energy reaches the brain. With unilateral placement this amount is much less, as the inter-electrode losses are greater with the electrodes being closer together. Therefore unilateral treatments should not be used with modern constant current apparatus, whose lower dosages can be relied on to keep side-effects to a minimum.

Unsatisfactory results from ECT can often be traced to inadequately trained operators who use minimal dosages and frequently have stimulus losses due to poor electrode-to-patient contact. The solution is to use an apparatus which will monitor the applied dosage, thus checking that a proper treatment has been given. Keeping a record of the individual dosages will give a cumulative total and make comparative results more accurate.

\section{Pasture Road \\ Letchworth \\ Herts SG6 $3 L S$}

R. J. RUSSELI

\section{Obstetric Complications and Schizophrenia}

SIR: Nimgaonkar et al (Journal, August 1988, 153, 191-197) did not find obstetric complications (OCs) to be commoner in non-familial than familial schizophrenia. In discussing their findings, the authors allude briefly to the possibility that OCs are epiphenomenia of schizophrenia rather than aetiological factors in their own right. This possibility needs to be taken seriously, since it can also explain why large geographical and class differences in perinatal mortality do not seem to lead to parallel differences in the rate of schizophrenia. While many authors have suggested that OCs produce an increased liability to schizophrenia, the existing evidence is more consistent with the notion that a liability to schizophrenia can result in OCs (Goodman, 1988).

It may seem ludicrous to suggest that a baby experiences more OCs because he or she is going to develop schizophrenia decades later. The theory is less far-fetched if schizophrenia is viewed as a neurodevelopmental disorder with different manifestations at different stages of the life cycle (Murray \& Lewis, 1987). Preschizophrenic children commonly manifest behavioural abnormalities, typically with social and attentional deficits (Rutter, 1985). If schizophrenia has different manifestations in adulthood and childhood, might it not have yet other manifestations in utero? The normal foetus is an active participant in preparations for delivery, and in the delivery process itself, which is presumably why pre-existent foetal abnormalities can result in birth complications (Nelson \& Ellenberg, 1986). It is at least conceivable that a single neuropathology, such as the presence of widespread neuronal misconnections (Randall, 1983), underlies impaired foetal participation in the birth process, childhood deficits in attentiveness and sociability, and adult psychosis.

Great Ormond Street Hospital

ROBERT GOODMAN

for Sick Children

London WCI 3JH

\section{References}

Goodman, R. (1988) Are complications of pregnancy and birth causes of schizophrenia? Developmental Medicine and Child Neurology, 30, 391-395.

MURRAY, R. M. \& LEWIS, S. W. (1987) Is schizophrenia a neuro-developmental disorder? British Medical Journal, 295, 681-682.

Nelson, K. B. \& Ellenberg, J. H. (1986) Antecedents of cerebral palsy: multivariate analysis of risk. New England Journal of Medicine, 315, 81-86.

RANDALL, P. L. (1983) Schizophrenia, abnormal connection, and brain evolution. Medical Hypotheses, 10, 247-280.

RutTer, M. (1985) Psychopathology and development: links between childhood and adult life. In Child and Adolescent Psychiatry: Modern Approaches (eds M. Rutter \& L. Hersov). Oxford: Blackwell. 